\title{
OPEN AND UNIFORMLY OPEN RELATIONS ${ }^{1}$
}

\author{
P. MAH AND S. A. NAIMPALLY
}

\begin{abstract}
It is shown that if $(X, \delta)$ is an Efremovic proximity space, $Y$ is a topological space, $R \subset X \times Y$ is an injective relation, then $R$ is open if and only if $R$ is weakly open, nearly open and $R[X]$ is open in $Y$. An analogous result is proved when $X$ is a uniform space and $Y$ a Morita uniform space: (i) if $R$ is uniformly open, then $R$ is weakly open and uniformly nearly open; (ii) if $R$ is weakly open, and uniformly nearly open, then $R$ is uniformly open on $X$ to $R[X]$. These results include, as special cases, results of Kelley, Pettis and Weston.
\end{abstract}

Our investigation began in an attempt to find a common refinement of a uniformly open mapping theorem of Kelley [1, Lemma 6.36], and an open mapping theorem of Weston [7, Theorem 8]. This problem was posed by Pettis [5] at the Topology Conference at Charlotte, N. C. In this paper, we prove two very general results concerning (uniform) openness of a relation (Theorems 3 and 7). From these two results, we derive generalizations of several known results. In particular, we derive generalizations of the results of Kelley and Weston, the former to Morita range space, and the latter to point-compact upper semicontinuous relations. This latter generalization of Weston's result allows us to both generalize (to relations) and dualize, in a certain sense, some recent results of Pettis. Finally, we give an example which shows that Weston's result cannot be generalized to a cocompact domain. This answers a question raised in [5].

The authors would like to thank the referee, Professors P. L. Sharma and A. J. Ward for several helpful comments and suggestions; especially the referee who made several creative suggestions.

Let $X$ and $Y$ be two topological spaces. We shall consider two types of proximities, Efremovič (or EF-proximity) and Lodato (or LO-proximity). Frequently one of the spaces considered is a metric space; in that case, if the metric is $d$, we shall suppose that the proximity considered is the EFproximity $\delta$ induced by $d$ viz. $A \delta B$ iff $d(A, B)=\inf \{d(a, b): a \in A$, $b \in B\}=0$. (Read $A \delta B$ as " $A$ is near $B$ "; its negation " $A$ is far from $B$ " is written as $A \delta B$.) Notations and results of [3] will be freely used in this paper.

\footnotetext{
Received by the editors July 10, 1974 and, in revised form, May 26, 1976.

AMS (MOS) subject classifications (1970). Primary 54C10, 54C60, 46A30; Secondary 54E05, 54E15.

Key words and phrases. Open relations, nearly open, weakly open, Morita uniformity, proximity, uniformly open, uniformly nearly open, nearly continuous, closed graph.

${ }^{1}$ The research of both authors was supported by operating grants from NRC of Canada.
} 
To obtain a generalization of Kelley's result, we shall assume the range space $Y$ of a relation to be an $R_{0}$-space (or weakly regular) i.e. $x \in\{y\}^{-}$ implies $y \in\{x\}^{-}$for all $x, y \in Y$, where ${ }^{-}$denotes the closure operator. For such a space, Morita [2] has shown that there is a family $\Omega$ (called a Morita uniformity) of open covers of $Y$ such that $\left\{\alpha^{*}(y): \alpha \in \Omega\right\}$ is a neighbourhood base at $y$ for each $y \in Y$, where $\alpha^{*}(y)=\bigcup\{G \in \alpha: y \in G\}$.

A relation $R \subset X \times Y$ is injective if $A \cap B=\varnothing$ in $X$ implies $R[A] \cap$ $R[B]=\varnothing$ in $Y$. We note that if $R$ is a function from $X$ to $Y$, the inverse relation $R^{-1}$ is always injective, and conversely, if $R$ is a relation on $X$ to $Y$ such that $R^{-1}$ is injective, then $R$ is a function. A relation is called bijective if it is injective and onto. A relation $R$ is called (i) open (resp. closed) iff for each open (resp. closed) set $A$ in $X, R[A]$ is open (resp. closed) in $Y$, (ii) nearly open iff for each open set $A$ in $X, R[A] \subset R[A]^{-0}$, (iii) upper semicontinuous (or USC) iff for each $x \in X$ and each open set $V$ in $Y$ such that $R(x) \subset V$, there is a neighbourhood $U$ of $x$ such that $R[U] \subset V$, (iv) nearly continuous (when $R$ is a function) iff for each open set $B$ in $Y, R^{-1}[B] \subset R^{-1}[B]^{-0}$, where ${ }^{0}$ denotes the interior operator. If $R$ is a closed subset of $X \times Y$, then we say that $R$ has a closed graph.

If $(X, \mathscr{Q})$ is a uniform space, and $(Y, \Omega)$ a Morita uniform space, then $R \subset X \times Y$ is called (i) uniformly open iff for each $U \in \mathcal{Q}$ there is an $\alpha \in \Omega$ such that for all $(x, y) \in R, \alpha^{*}(y) \subset R[U[x]]$, (ii) uniformly nearly open iff for each $U \in \mathcal{Q}$, there is an $\alpha \in \Omega$ such that for all $(x, y) \in R, \alpha^{*}(y) \subset$ $R[U[x]]^{-0}$. If $(X, \delta)$ is a LO-proximity space, then $R \subset X \times Y$ is called weakly open iff for each $A \subset X, y \in R[A]^{-} \cap R[X]$ implies $R^{-1}(y) \delta A$ (see Poljakov [6]). (For our purposes, it is sufficient that the above holds for each open set $A$.)

It is obvious that a (uniformly) open relation is (resp. uniformly) nearly open. However, contrary to an assertion in [6], an open relation need not be weakly open as the following example shows.

1. Example. Let $X$ be the subset of $\mathbf{R}^{2}$ defined by $\{(x, y): y>5\} \cup$ $\{(x, y): x>0$ and $y>1\}$, with the subspace proximity $\delta$ induced by the usual Euclidean metric. Let $f: X \rightarrow \mathbf{R}$ be the projection $f(x, y)=x$. Since $X$ is open in $\mathbf{R}^{2}, f$ is an open map. If $A=\{(x, y): x>0,1<y<2\}$ then $0 \in f(A)^{-}$ but $f^{-1}(0)=\{(0, y): y>5\} 8 A$. Thus $f$ is not weakly open.

If we require the relation to be injective, then the assertion of [6] is true.

2. Lemma. If $(X, \delta)$ is a LO-proximity space, $Y$ a topological space, and $R \subset X \times Y$ is an open injective relation, then $R$ is weakly open.

Proof. If the result is not true, then there exist $A \subset X, y \in R[A]^{-} \cap$ $R[X]$ such that $x \notin A^{-}$, where $x=R^{-1}(y)$ (noting that $R^{-1}$ is a function since $R$ is injective). Thus, there is an open set $U$ in $X$ such that $x \in U$ and $U \cap A=\varnothing$. Since $R$ is injective and open, $R[U]$ is an open set containing $y$ and disjoint from $R[A]$, a contradiction. 
In the opposite direction, we have the following fundamental result of this paper.

3. THEOREM. If $(X, \delta)$ is an EF-proximity space, $Y$ is a topological space, $R \subset X \times Y$ is a weakly open and nearly open relation, then $R$ is open on $X$ to $R[X]$.

Proof. We must show that $R[V]$ is open in $R[X]$ for each open set $V$ in $X$. Let $y \in R[V]$. Then there is an $x \in V$ with $(x, y) \in R$. Since $x \delta(X \sim V)$, there is a neighbourhood $U$ of $x$ with $U \delta(X \sim V)$. Since $R$ is nearly open, $R[U]^{-}$is a neighbourhood of $y$, and so the proof is complete once we show that $R[U]^{-} \cap R[X] \subset R[V]$. Suppose, then, that $z \in R[U]^{-} \cap R[X]$. Since $R$ is weakly open, $R^{-1}(z) \delta U$, and since $U \delta(X \sim V)$, we have $R^{-1}(z) \cap V \neq \varnothing$. Thus $z \in R[V]$.

We note that if $R[X]$ is closed, then it is also open, since $R[X] \subset R[X]^{-0}$ $=R[X]^{0}$. Thus, if $R[X]$ is either open or closed in $Y$, then under the hypothesis of Theorem $3, R$ is open.

4. Corollary. If $(X, \delta)$ is an EF-proximity space, $Y$ is a topological space, $R \subset X \times Y$ is an injective relation, then $R$ is open iff $R$ is weakly open, nearly open and $R[X]$ is open.

We now derive a generalized version of a well-known result of Weston [7, Theorem 8]. A relation $R \subset X \times Y$ is called separating iff for each pair $x_{1}, x_{2}$ of distinct points of $X$, there exist neighbourhoods $U_{1}, U_{2}$ respectively such that $R\left[U_{1}\right]^{-} \cap R\left[U_{2}\right]=\varnothing=R\left[U_{1}\right] \cap R\left[U_{2}\right]^{-}$. Clearly if $R$ is separating and the domain of $R$ is $X$ then $X$ is Hausdorff. Also, if $R$ is separating, then $R$ is injective and $R(x)$ is closed in $R[X]$ for all $x$. Conversely, any one of the following conditions is sufficient for $R$ to be separating:

(a) $X$ is Hausdorff and $R$ is open and injective;

(b) $Y$ is Hausdorff, $R$ is USC, injective and point-compact;

(c) $Y$ is $T_{4}, R$ is USC and injective.

When applied in case (b), the next theorem clearly yields Weston's result [7, Theorem 8].

5. THEOREM. If $(X, d)$ is a complete metric space, $R \subset X \times Y$ is separating, nearly open and $R[X]$ is open or closed in $Y$, then $R$ is open.

Proof. In view of the remarks immediately following Theorem 3, it suffices to prove the theorem when $R[X]$ is open. Our proof is patterned after Weston's proof in [7]. By Corollary 4, it is sufficient to prove that $R$ is weakly open. Suppose then that $A \subset X, y \in R[A]^{-} \cap R[X], q_{0}=R^{-1}(y)$ (a single point as $R$ is injective). We must show that $q_{0} \in A^{-}$. Take any $r_{0}>0$ and put $r_{m}=r_{0} / 2^{m}$. Suppose that, for a given $n \geqslant 0, q_{m}$ has been defined for all $m, 0 \leqslant m \leqslant n$, and $x_{m}$ for $0 \leqslant m<n$ such that the following conditions are satisfied:

(i) $x_{m} \in U\left(x_{m-1}, r_{m}\right)$ for $1 \leqslant m<n$; 
(ii) $q_{m} \in U\left(q_{m-1}, r_{m-1}\right)$ for $1 \leqslant m \leqslant n$;

(iii) $R\left[U\left(q_{m}, r_{m}\right)\right]^{-} \cap R\left[A_{m}\right] \neq \varnothing$ for $0 \leqslant m \leqslant n$, where $A_{0}=A$ and $A_{m}=U\left(x_{m-1}, r_{m}\right)$ for $1 \leqslant m \leqslant n$.

(For $n=0$, (i) and (ii) are vacuous, and (iii) is satisfied because, $R$ being nearly open, $R\left[U\left(q_{0}, r_{0}\right)\right]^{-}$is a neighbourhood of $R\left(q_{0}\right)$ and so of $y$, and hence intersects $R[A]$.)

Then by (iii), we can choose for $n \geqslant 0, x_{n} \in A_{n}$ with $R\left(x_{n}\right) \cap R\left[U\left(q_{n}\right.\right.$, $\left.\left.r_{n}\right)\right]^{-} \neq \varnothing$. This shows that (i) is satisfied for $m=n>0$. Again $R\left[U\left(x_{n}\right.\right.$, $\left.\left.r_{n+1}\right)\right]^{-}$is a neighbourhood of $R\left(x_{n}\right)$ and hence intersects $R\left[U\left(q_{n}, r_{n}\right)\right.$ ]. So we can choose $q_{n+1} \in U\left(q_{n}, r_{n}\right)$ with $R\left(q_{n+1}\right) \cap R\left[U\left(x_{n}, r_{n+1}\right)\right]^{-} \neq \varnothing$. Thus (ii) is satisfied for $m=n+1$. A similar application shows that (iii) is also satisfied for $m=n+1$. Hence the construction is possible inductively for all $m$. Clearly, $\left(x_{m}\right)$ and $\left(q_{m}\right)$ form Cauchy sequences with respective limits $x, q$ say. We claim that $x=q$; for if not, neighbourhoods $N_{x}, N_{q}$ would exist as in the definition of a separating relation, and for sufficiently large $m, U\left(q_{m}\right.$, $\left.r_{m}\right) \subset N_{q}$ and $U\left(x_{m-1}, r_{m}\right) \subset N_{x}$, violating condition (iii). By construction $d\left(q, q_{0}\right) \leqslant 2 r_{0}$ and $d\left(x, x_{0}\right) \leqslant r_{0}$; so as $x_{0} \in A$ and $r_{0}$ is arbitrary, we have $q_{0} \in A^{-}$as required.

If $f: X \rightarrow Y$ is a function, then $f$ is closed iff $f^{-1}$ is USC; thus taking $R=f^{-1}$ in the above result we get the following.

6. Corollary. Let $X$ be $T_{2}$ (resp. $T_{4}$ ), $Y$ a complete metric space, and $f$ : $X \rightarrow Y$ a nearly continuous, closed function with compact (resp. no condition) fibers. Then $f$ is continuous.

We now consider uniformly (nearly) open relations in order to generalize Kelley's result, which is done in 8.

7. Theorem. Let $(X$, Q $)$ be a uniform space, and $(Y, \Omega)$ be an $R_{0}$ Morita uniform space.

(i) If $R$ is uniformly open, then $R$ is weakly open and uniformly nearly open.

(ii) If $R$ is weakly open and uniformly nearly open, then $R$ is uniformly open on $X$ to $R[X]$.

Thus it follows that $R$ is uniformly open iff $R$ is weakly open, uniformly nearly open and $R[X]$ is either open or closed.

Proof. (i) Suppose $R$ is uniformly open. We must show that $R$ is weakly open; if this is false then there exist $A \subset X, y \in R[A]^{-} \cap R[X], U \in$ Q such that $U[A] \cap B=\varnothing$ where $B=R^{-1}(y)$. Thus, $R[U[A]] \cap R[B]=$ $R[U[A]] \cap\{y\}=\varnothing$ (for otherwise, $y \in R[U[A]]$ and so $B \cap U[A] \neq \varnothing$, a contradiction). Since $R$ is uniformly open, there exists an $\alpha \in \Omega$ such that for all $(x, y) \in R, \alpha^{*}(y) \subset R[U[x]]$, and so $\alpha^{*}(R[A]) \subset R[U[A]]$, where $\alpha^{*}(Z)$ $=\bigcup\left\{\alpha^{*}(z): z \in Z\right\}$. Thus, $y \notin \alpha^{*}(R[A])$. Since $Y$ is $R_{0}, \alpha^{*}(y) \cap R[A]=$ $\varnothing$, contradicting $y \in R[A]^{-}$. Clearly $R$ is uniformly nearly open if it is uniformly open.

(ii) Assume $R$ is uniformly nearly open and weakly open. The former 
property assures us that for every $U \in \mathcal{Q}$, there is an $\alpha \in \Omega$ such that for all $(x, y) \in R, \alpha^{*}(y) \subset R[V[x]]^{-}$, where $V \in \mathcal{Q}$ with $V^{2} \subset U$. To prove $R$ is uniformly open in $R[X]$, we show $R[V[x]]^{-} \cap R[X] \subset R[U[x]]$. Suppose then $z \in R[V[x]]^{-} \cap R[X]$. Since $R$ is weakly open, $R^{-1}(z) \delta V[x]$. But $V[x] 8(X \sim U[x])$, and so $R^{-1}(z) \cap U[x] \neq \varnothing$ i.e. $z \in R[U[x]]$.

8. Corollary. Let $(X, d)$ be a complete metric space, $(Y, \Omega)$ an $R_{0}$ Morita uniform space, and $R \subset X \times Y$ a relation with a closed graph. If $R$ is uniformly nearly open, then $R$ is uniformly open.

Proof. This proof is patterned after Kelley [1, Lemma 6.36]. It is sufficient to show that for any subset $A \subset X$, if there is a $p \in Y$ such that $p \in R[A]^{-}$, then $R^{-1}(p) \delta A$. This will not only show that $R$ is weakly open, but also that $R[X]$ is closed in $Y$. This latter implication follows by choosing $A=X$, and noting that, if $p \in R[X]^{-}$, then $R^{-1}(p) \delta X$ and so $R^{-1}(p) \neq \varnothing$. Since $R$ is uniformly nearly open, it then follows that $R[X]$ is open; Theorem 7 then can be applied to conclude that $R$ is uniformly open.

Let, then, $A \subset X, p \in R[A]^{-}$, and $\varepsilon>0$. There is an $\alpha \in \Omega$ such that for all $(x, y) \in R, \alpha^{*}(y) \subset R\left[U\left(x, \varepsilon / 2^{2}\right)\right]^{-}$. Since $p \in R[A]^{-}, \alpha^{*}(p) \cap R[A] \neq$ $\varnothing$, and so there exists $(u, v) \in R$ such that $u \in A$ and $v \in \alpha^{*}(p)$. Then $p \in \alpha^{*}(v) \subset R\left[U\left(u, \varepsilon / 2^{2}\right)\right]^{-}$. We now choose inductively a sequence of subsets $A_{n}, n \geqslant 0$, with the properties, diameter of $A_{n}<\varepsilon / 2^{n}$ (for $n>0$ ), $A_{n} \cap A_{n-1} \neq \varnothing$, and $p \in R\left[A_{n}\right]^{-}$as follows: Set $A_{0}=A, A_{1}=U\left(u, \varepsilon / 2^{2}\right)$. The step from $A_{n-1}$ to $A_{n}, n>1$, is to choose $\alpha_{n} \in \Omega$ such that for all (x, $y) \in R, \alpha_{n}^{*}(y) \subset R\left[U\left(x, \varepsilon / 2^{n+1}\right)\right]^{-}$. Then since $p \in R\left[A_{n-1}\right]^{-}, \alpha_{n}^{*}(p) \cap$ $R\left[A_{n-1}\right] \neq \varnothing$, and so there exists $\left(u_{n}, v_{n}\right) \in R$ such that $u_{n} \in A_{n-1}$ and $v_{n} \in \alpha_{n}^{*}(p)$. Consequently, $p \in \alpha_{n}^{*}\left(v_{n}\right) \subset R\left[U\left(u_{n}, \varepsilon / 2^{n+1}\right)\right]^{-}$. Set $A_{n}=U\left(u_{n}\right.$, $\left.\varepsilon / 2^{n+1}\right)$. If $x_{n} \in A_{n} \cap A_{n-1}$ for $n \geqslant 1$, then $\left(x_{n}\right)$ is a Cauchy sequence, and since $X$ is complete, it converges to $x \in X$. Clearly $d(x, u)<\varepsilon$. We now show that $(x, p) \in R$. If $W$ is any neighbourhood of $x$, then $W$ contains some $A_{n}$, and so $p \in R[W]^{-}$. If $V$ is any neighbourhood of $p$, then $V \cap R[W] \neq$ $\varnothing$, and so there is an $s \in W, t \in V$ with $(s, t) \in R$. This shows that $R \cap(W \times V) \neq \varnothing$, and since $R$ has a closed graph, $(x, p) \in R$. Thus $R^{-1}(p) \delta A$.

We now prove an analogue of the above for uniformly locally compact space [1, p. 214].

9. Corollary. Let $(X, \mathcal{Q})$ be a uniformly locally compact space (i.e. there is a $U_{0} \in \mathcal{Q}$ such that $U_{0}[x]$ is compact for all $\left.x \in X\right),(Y, \Omega)$ be a Morita uniform space, $R \subset X \times Y$ be a graph-closed uniformly nearly open relation. Then $R$ is uniformly open.

Proof. It is sufficient to show that if $A \subset X, p \in R[A]^{-}$, then $R^{-1}(p) \delta A$. This will show that $R$ is weakly open and $R[X]$ is closed. Thus by Theorem 7, it will follow that $R$ is uniformly open.

Suppose then, that there exist $A \subset X, p \in R[A]^{-}$such that $R^{-1}(p) \& A$. 
Let $U_{0} \in \mathcal{Q}$ be such that $U_{0}[x]$ is compact for all $x$. Choose a symmetric $U \in \mathcal{Q}$ such that $U^{2} \subset U_{0}$ and $R^{-1}(p) \cap U^{2}[A]=\varnothing$. Since $R$ is uniformly nearly open, there is an $\alpha_{1} \in \Omega$ such that for all $x \in X, \alpha_{1}^{*}(R(x)) \subset$ $R[U[x]]^{-0}$. Since $p \in R[A]^{-} \subset \alpha_{1}^{*}(R[A])$, we can choose $x_{1} \in A$ such that $p \in \alpha_{1}^{*}\left(R\left(x_{1}\right)\right) \subset R\left[U\left[x_{1}\right]\right]^{-}$. Now $U\left[x_{1}\right]^{-}$is compact and does not meet $R^{-1}(p)$, i.e. $R \cap\left(U\left[x_{1}\right]^{-} \times\{p\}\right)=\varnothing$. For each $x \in U\left[x_{1}\right]^{-}$, we can find a $U_{x} \in \mathscr{Q}, \alpha_{x} \in \Omega$ such that $R \cap\left(U_{x}[x] \times \alpha_{x}^{*}(p)\right)=\varnothing$. Since $U\left[x_{1}\right]^{-}$is compact, it can be covered by finitely many $U_{x}[x]$, and hence we can find an $\alpha \in \Omega$ such that $R \cap\left(U\left[x_{1}\right]^{-} \times \alpha^{*}(p)\right)=\varnothing$. But this contradicts $p \in$ $R\left[U\left[x_{1}\right]\right]^{-}$.

We now show how our results are related to some recent results of Pettis [4].

10. Theorem. Suppose $Y$ is Hausdorff, $R \subset X \times Y$ is a bijective relation with a metrically complete graph. If $R$ is nearly open, then $R$ is open.

Proof. Let $P_{Y}$ (resp. $P_{X}$ ) be the projection from $X \times Y$ onto $Y$ (resp. $X$ ), and $P_{R Y}$ (resp. $P_{R X}$ ) be the restriction of $P_{Y}$ (resp. $P_{X}$ ) to $R$. Then since $R$ is bijective, $P_{R Y}$ is bijective. Moreover, $P_{R Y}$ is continuous. Since $R=$ $P_{R Y} \circ P_{R X}^{-1}$, our result is proved if we show that $P_{R Y}$ is nearly open. Let $V$ be open in $R$ and $y_{0} \in P_{R Y}(V)$. There exist open sets $U_{X}, U_{Y}$ in $X, Y$ respectively, such that $\left(U_{X} \times U_{Y}\right) \cap R \subset V, R^{-1}\left(y_{0}\right) \in U_{X}, y_{0} \in U_{Y}$. Then $y_{0} \in$ $R\left[U_{X}\right] \cap U_{Y} \subset P_{R Y}(V)$. Since $R\left[U_{x}\right] \subset R\left[U_{x}\right]^{-0}, y_{0} \in R\left[U_{x}\right]^{-0}$. But as $U_{Y}$ is open, $U_{Y} \cap R\left[U_{x}\right]^{-0}=U_{Y} \cap\left(R\left[U_{x}\right] \cap U_{Y}\right)^{-0}$. Thus $y_{0} \in P_{R Y}(V)^{-0}$, and the result is proved.

Since a closed subset of a metrically complete space is metrically complete we get from the above,

11. Corollary. If $X$ and $Y$ are metrically complete, $R \subset X \times Y$ is a bijective nearly open relation with a closed graph, then $R$ is open.

Theorem 10 and Corollary 11 are the relation form of (IV) and (V) in Pettis [4]. Under appropriate hypotheses on the spaces and on the function $f$ : $X \rightarrow Y$, we can apply Theorem 10 to $f^{-1}$ to yield (I) of [4], and Corollary 11 to $f^{-1}$ to yield (II) in [4].

Finally, we answer a question of Pettis [5] by showing that Weston's result does not generalize to cocompact domain.

12. EXAMPLE. Let $X=\mathbf{R}$ with the topology having as subbase the family of all open intervals together with the rationals. Then $X$ is a Hausdorff cocompact space [8, p. 10]. Let $Y=\mathbf{R}$ with the usual topology. Then the identity map from $X$ to $Y$ is a continuous nearly open bijection but is not open.

Added in revision. The referee suggested the following conjecture which includes our Theorem 5 as well as the one recently proved by T. Byczkowski and R. Pol (On the closed graph and open mapping theorems, Bull. Acad. Polon. Sci. 24 (1976), 723-726). 
Conjecture. Let $X$ be regular and strongly countably complete, $Y$ a topological space, $R \subset X \times Y$ a nearly open relation from $X$ to $Y$, with $R[X]$ either open or closed in $Y$. Then $R$ is open from $X$ to $Y$ if either (i) $R$ is separating, or (ii) $R$ has a closed graph, $R$ is injective, and $R[A]$ is compact whenever $A$ is compact.

We are unable to prove the above conjecture. The following result is an improvement of our Theorem 5, but is independent of the result due to Byczkowski and Pol (loc cit). We observe that in our Theorem 3 we need $\delta$ to be only an $R$-proximity (see D. Harris, Regular-closed spaces and proximities, Pacific J. Math. 34 (1970), 675-685). For unfamiliar terms in the following see J. M. Aarts and D. J. Lutzer, Completeness properties designed for recognizing Baire spaces, Dissertationes Math. 116 (1974), 48 pp.

The proof of the following theorem is similar to that of T. Byczkowski and R. Pol (loc cit).

TheOREM. Let $X$ be a Rudin-complete Moore space, $Y$ a topological space, $R \subset X \times Y$ a nearly open separating relation from $X$ to $Y$ with $R[X]$ either open or closed in $Y$. Then $R$ is open from $X$ to $Y$.

Proof. As in the proof of Theorem 5, it suffices to prove that $R$ is weakly open when $R[X]$ is open. To this end, we first prove that for any open set $U$ in $X, R[U]^{-} \subset R\left[U^{-}\right]$. Let $\{\mathcal{G}(n)\}$ be a nested development for $X$ which is Rudin-complete. If $R[U]^{-} \not R\left[U^{-}\right]$, then $R[U]^{-} \cap R[V] \neq \varnothing$, where $V$ $=X \sim U^{-}$. Take $y_{1} \in R[U]^{-} \cap R[V]$ and $x_{1} \in R^{-1}\left(y_{1}\right) \cap V$. Let $n_{1}$ be such that

$$
\operatorname{St}\left(x_{1}, \mathcal{G}\left(n_{1}\right)\right)=\bigcup\left\{G: x_{1} \in G \in \mathcal{G}\left(n_{1}\right)\right\} \subset V,
$$

and let $G_{1} \in \mathcal{G}\left(n_{1}\right)$ be such that $x_{1} \in G_{1} \subset V$. Since $R\left[G_{1}\right]^{-}$is a neighbourhood of $y_{1}, R\left[G_{1}\right]^{-} \cap R[U] \neq \varnothing$; hence there is a $z_{1} \in R\left[G_{1}\right]^{-} \cap$ $R[U]$ and $u_{1} \in R^{-1}\left(z_{1}\right) \cap U$. Let $m_{1}$ be such that $\operatorname{St}\left(u_{1}, \mathcal{G}\left(m_{1}\right)\right) \subset U$ and $H_{1} \in \mathcal{G}\left(m_{1}\right)$ be such that $u_{1} \in H_{1} \subset U$. Now choose inductively two sequences $\left\{n_{i}\right\}$ and $\left\{m_{i}\right\}$ such that

(i) $G_{i} \in \mathcal{G}\left(n_{i}\right), H_{i} \in \mathcal{G}\left(m_{i}\right)$;

(ii) $R\left[H_{i}\right]^{-} \cap R\left[G_{i}\right] \neq \varnothing$; and

(iii) $G_{i+1}^{-} \subset G_{i}, H_{i+1}^{-} \subset H_{i}$.

((iii) follows from the fact that a Moore space is regular.)

Since $X$ is Rudin-complete, $A_{0}=\cap\left\{H_{i}: i \geqslant 1\right\}$ and $A_{1}=\cap\left\{G_{i}: i \geqslant 1\right\}$ are nonvoid disjoint sets. Let $x_{i} \in A_{i}$ for $i=0,1$. Since $R$ is separating, there are neighbourhoods $N_{i}$ of $x_{i}, i=0,1$, such that $R\left[N_{0}\right]^{-} \cap R\left[N_{1}\right]=\varnothing$. Since $\{\mathcal{G}(n)\}$ is a development, there is an $n$ such that $H_{n} \subset N_{0}$ and $G_{n} \subset N_{1}$ and so $R\left[H_{n}\right]^{-} \cap R\left[G_{n}\right]=\varnothing$, a contradiction. Thus $R[U]^{-} \subset R\left[U^{-}\right]$for every open set $U$ in $X$.

Now it follows that $R$ is weakly open. Suppose $A \subset X, y \in R[A]^{-} \cap$ $R[X]$ and $q=R^{-1}(y)$. If $q \notin A^{-}$then there is an open neighbourhood $U$ of $q$ such that $U^{-} \cap A^{-}=\varnothing$. Since $R$ is injective, $R\left[U^{-}\right] \cap R\left[A^{-}\right]=\varnothing$. But 
$R[U]^{-} \subset R\left[U^{-}\right]$, and hence $R[U]^{-} \cap R[A]=\varnothing$, a contradiction.

REMARKS.

(i) For a Moore space, Rudin-completeness is equivalent to subcompactness.

(ii) There is a completely regular Moore space $X$ which is Rudin complete but not Čech complete.

\section{REFERENCES}

1. J. L. Kelley, General topology, Van Nostrand, Princeton, N. J., 1955.

2. K. Morita, On the simple extension of a space with respect to a uniformity. I, Proc. Japan Acad. 27 (1951), 65-72.

3. S. A. Naimpally and B. D. Warrack, Proximity spaces, Cambridge Univ. Press, Cambridge, 1970.

4. B. J. Pettis, Closed graph and open mapping theorems in certain topologically complete spaces, Bull. London Math. Soc. 6 (1974), 37-41.

5. Some topological questions related to open and closed graph theorems, Studies in Topology, Academic Press, New York, 1975, pp. 451-456.

6. V. Z. Poljakov, Open mappings of proximity spaces, Soviet Math. Dokl. 5 (1964), 545-548.

7. J. D. Weston, On the comparison of topologies, J. London Math. Soc. 32 (1957), 342-354.

8. Colloquium Co-topology 1964-1965, Mathematical Centre (Amsterdam) [Notes by J. M. Aarts].

Department of Mathematical Sciences, Lakehead University, Thunder Bay 'P', Ontario, Canada P7B 5E1 